\title{
Evaluation of Organ-At-Risk (OAR) Sparing in Vaginal Cuff Boost Treatment for Endometrial Cancer using Stereotactic Body Radiotherapy (SBRT) and Brachytherapy
}

\author{
*Selcuk Demiral, Omer Sager, Ferrat Dincoglan, Hakan Gamsiz, Bora Uysal, Esin Gundem, BaharDirican and \\ Murat Beyzadeoglu
}

Department of Radiation Oncology, University of Health Sciences, Turkey

Submission: October 03, 2017; Published: October 23, 2017

"Correspondence Address: Selcuk Demiral, Associate Professor, University of Health Sciences, Gulhane Medical Faculty, Department of Radiation Oncology, Gn. Tevfik Saglam Cad. 06018, Etlik, Kecioren, Ankara, Turkey, Tel: +90 312304 4689; Fax: +90 312 304 4680; Email:drs.demiral@hotmail.com

Abstract

Purpose: The aim of this study is to evaluate Organ-At-Risk (OAR) sparing in vaginal cuff boost treatment for endometrial cancer by making a dosimetric comparison between Stereotactic Body Radiotherapy (SBRT) and Brachytherapy modalities.

Material and Methods: A total of 18 patients with stage I-III endometrial cancer who underwent total abdominal hysterectomy and bilateral salpingo-oophorectomy \pm pelvic/paraortic lymphadenectomy followed by external pelvic radiotherapy and LINAC-based Stereotactic Body Radiotherapy boost therapy were enrolled. For the vaginal cuff boost treatment, SBRT and 2-D conventional brachytherapy plannings were compared with respect to target and critical organ point doses.

Results: There was a statistically significant reduction in bladder and rectum point doses with SBRT compared to Brachytherapy planning (bladder $\mathrm{p}<0.001$, rectum $\mathrm{p}<0.001$ ) whereas target point doses were equal with both modalities.

Conclusion: The use of SBRT modality conferred improved OAR sparing in vaginal cuff boost treatment for endometrial cancer without comprimising target point doses. Superior OAR sparing with SBRT may have implications for potential dose escalation to improve treatment outcomes in endometrial cancer management despite the need for futher supporting evidence.

Keywords: Endometrial cancer; Stereotactic body radiotherapy;Brachytherapy; Vaginal cuff boost

Abbreviations: OAR:Organ-At-Risk; SBRT: Stereotactic Body Radiotherapy

\section{Introduction}

Postoperative radiotherapy (RT) followed by vaginal cuff brachytherapy boost is used to prevent recurrences in the vaginal cuff in endometrial cancer management [1]. 8-year vaginal cuff local control rates for patients with endometrial carcinoma were reported to be $85 \%$ with surgery alone, $96 \%$ with adjuvant pelvic RT alone and combined pelvic RT + brachytherapy has yielded a local control rate of $97.3 \%$ in the studies by Creutzberg et al. \& Mandell et al. [2,3]. While improving local control, postoperative vaginal cuff boost radiotherapy may pose the risk of treatment related toxicity due to intimate association of critical organs with the high-dose region [4]. In the study by Macleod et al., complication rates were reported to be in the range of 8-23\% and $25-45 \%$ with postoperative vaginal vault brachytherapy alone and combined external pelvic RT + brachytherapy, respectively [5].

Given the longer life expectancy in endometrial cancer patients with recent advances in local and systemic therapeutic approaches, quality-of-life has gained utmost priority and improving the toxicity profile of radiation deliveryto reduce RT induced toxicity has become an indispensable aspect of current radiotherapeutic management.In this context, SBRT has emerged as a therapeutic option to deliver vaginal cuff boost in endometrial cancer patients with promising results [6,7]. In this study, we evaluated target and critical organ doses with conventional brachytherapy and SBRT in our patients receiving vaginal cuff boost after external pelvic RT for endometrial cancer. 


\section{Cancer Therapy \& Oncology International Journal}

\section{Material and Methods}

A total of 18 endometrial cancer patients were enrolled in this ethics committee-approved study.Written informed consent was obtained from all patients and all procedures were in accordance with the Helsinki Declaration of 1975, as revised in 2000. After complete surgical staging with TAH+BSO and PPLND, all patients received postoperative pelvic RT of 45 Gy in 25 fractions followed by Linear Accelerator (LINAC)-based SBRT boost. For the purpose of this study, all patients also underwent 2-D conventional brachytherapy planning to compare with SBRT boost planning. Target, bladder, and rectum point doses were used for dosimetric comparison between the two modalities. Total vaginal cuff boost dose was 18 Gy deliveredin 3 fraction sat 1-week intervals.

In treatment simulation for SBRT, all patients were positioned in supine position with arms on the chest using customized vacuum body (BlueBAG, Bodyfix system, Medizintechnik GmbH, Germany) for immobilization. Initially, foley catheter was inserted and foley balloon was inflated with 7 cc radiopaque contrast. Vaginal radio-opaque device (highdose-rate Brachytherapy cylinder) was used to visualizethe CTV2 as the upper two thirds of the vagina at CT simulation(CT Lightspeed, GE Healthcare, Chalfont St. Giles, UK). After fixation of Bodyfix localizer (Medizintechnik $\mathrm{GmbH}$, Germany) to CT Simulator, cross-sectional images with $1.25 \mathrm{~mm}$ slice thickness were acquired.

The acquired treatment planning CT images were sent to the contouring workstation via miniPACS system. Advantage SimMD simulation and localization software (Advantage SimMD, GE, UK) was used to contour the CTV and Organs-At-Risk (OARs). CTV2 was contoured to define the upper two thirds of vagina (approximately $4 \mathrm{~cm}$ ). CTV2 was isotropically expanded by 5-mm to generate the PTV2. All 18 patients were treated with the dynamic-arc technique using a single isocenter and 9-17 arcs. Dose was prescribed at a distance of $0.5 \mathrm{~cm}$ from the cylinder surface. Total prescribed dose was 18 Gydelivered in 3 fractions of 6 Gy at 1-week intervals. ERGO treatment planning system (ERGO + +, Elekta, UK) and dynamic conformal arc technique was used in treatment planning.

After CT-simulation for SBRT, rectal barium was administered and anteroposterior and lateral radiographs were taken for 2-D conventional brachytherapy planning (Nucletron Plato planning system). Bladder and rectum point doses were recorded according to International Commission on Radiation Units and Measurements (ICRU-38). Bladder, rectum, and target point doses in SBRT and 2-D conventional brachytherapy plannings were compared. Wilcoxon test was used to compare bladder and rectum point doses for OAR sparing with SBRT and brachytherapy modalities, and $\mathrm{p}<0.05$ was considered as the level of statistical significance.

\section{Results}

SBRT and brachytherapy boost treatment plannings were compared with respect to target and critical organ doses for all 18 endometrial cancer patients. Median age was 62 (53-65) years. Histopathological diagnosis was adenocarcinoma in all 18 patients. Out of the total 18 patients, 10 patients had stage IB, 6 patients had stage II, and 2 patients had stage IIIC1 endometrial cancer according to American Joint Committee on Cancer staging (AJCC 2010). All patients initially received pelvic RT to clinical target volume (CTV1) (pelvic lymph nodes, tumor bed) with a 4 -field box technique in the supine position. All fields were treated daily, 5 days a week, with 6-18 MV X-rays. SBRT was given to CTV2 (vaginal cuff) boost site using dynamic-arc techniques with a commercially available multileaf collimatorbased LINAC treatment machine (Elekta Synergy, UK ) and 6-MV $\mathrm{X}$-rays was used in this stereotactic setting.

Table 1: Bladder and rectum point doses SBRT and Brachytherapy (cGy).

\begin{tabular}{|c|c|c|c|c|}
\hline $\begin{array}{c}\text { Patient } \\
\text { No }\end{array}$ & $\begin{array}{c}\text { SBRT } \\
\text { Bladder }\end{array}$ & $\begin{array}{c}\text { Brachytherapy } \\
\text { Bladder }\end{array}$ & $\begin{array}{c}\text { SBRT } \\
\text { Rectum }\end{array}$ & $\begin{array}{c}\text { Brachytherapy } \\
\text { Rectum }\end{array}$ \\
\hline 1 & 522 & 2295 & 1292 & 3123 \\
\hline 2 & 1002 & 1800 & 1731 & 1998 \\
\hline 3 & 424 & 2286 & 1620 & 2601 \\
\hline 4 & 90 & 1386 & 1017 & 1827 \\
\hline 5 & 851 & 1980 & 1728 & 2106 \\
\hline 6 & 1211 & 3096 & 1697 & 1665 \\
\hline 7 & 255 & 1834 & 1594 & 2018 \\
\hline 8 & 360 & 2019 & 1488 & 1975 \\
\hline 9 & 144 & 2256 & 1580 & 2745 \\
\hline 10 & 552 & 2140 & 1629 & 2140 \\
\hline 11 & 379 & 1305 & 1450 & 2199 \\
\hline 12 & 411 & 1587 & 1457 & 2604 \\
\hline 13 & 756 & 1834 & 1346 & 1967 \\
\hline 14 & 218 & 2183 & 1711 & 1834 \\
\hline 15 & 287 & 2021 & 1301 & 2697 \\
\hline 16 & 170 & 1543 & 1145 & 2704 \\
\hline 17 & 564 & 1480 & 1203 & 1894 \\
\hline 18 & 864 & 1987 & 1380 & 2507 \\
\hline & & & & \\
\hline
\end{tabular}

In both SBRT boost and brachytherapy boost treatment plans, target point doses were calculated equal as 600 cGy. Median point dose measured to compare normal tissue sparing for bladder in SBRT and brachytherapy plannings was 503 cGy \pm 318.3 cGy (90 cGy-1211 cGy) and $1946.2 \pm 423$ cGy (1305 cGy-3096 cGy) respectively revealing statistically significant reduction in bladder point dose with SBRT compared to brachytherapy boost planning $(\mathrm{p}<0.001)$. Rectal point dose in SBRT and brachytherapy plannings was 1464.9 cGy \pm 215 cGy (1017 cGy-1731 cGy) and2255.7 cGy \pm 409.9 cGy (1665 


\section{Cancer Therapy \& Oncology International Journal}

cGy-3123 cGy) respectively which also revealed statistically significant reduction inrectal point dose with SBRT compared to brachytherapy boost planning $(\mathrm{p}<0.001)$. Table 1 shows bladder and rectum point doses for SBRT and Brachytherapy.

Figure 1 shows sagittal treatment planning images of a patient treated with vaginal cuff SBRT using vaginal cylindirical applicator.

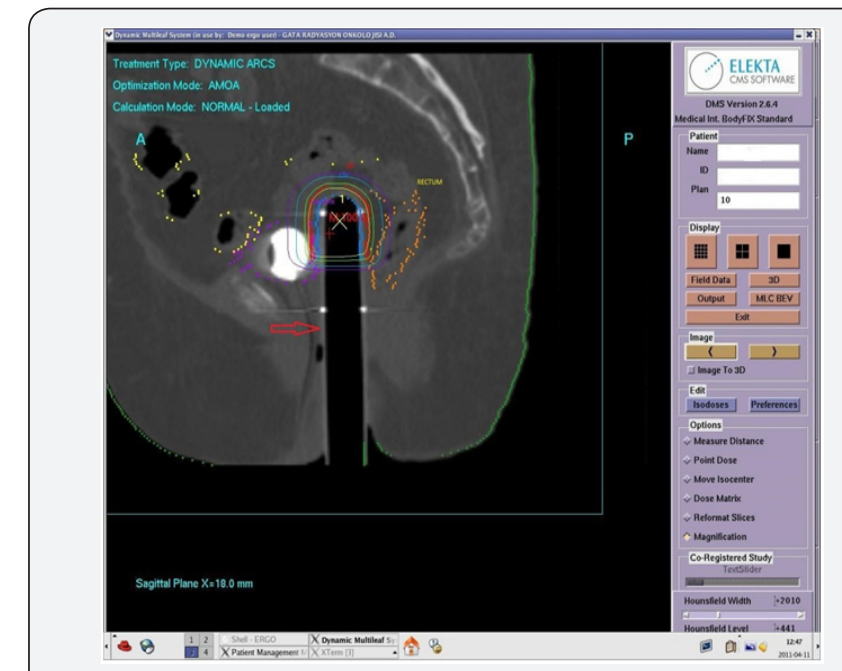

Figure 1: Sagittal treatment planning images of a patient treated with vaginal cuff SBRT using vaginal cylindirical applicator (red arrow showing the vaginal cylinder).

\section{Discussion}

In the management of endometrial cancers, vaginal cuff RT with or without external pelvic RT improves local control in the postoperative setting and is used as an adjunct to surgery [3]. Nevertheless, surgery hampers ther epair mechanism through decreasing the blood flow resulting in increased RT toxicity [8]. As the parametrial tissueis removed in patients undergoing hysterectomy, the rectum and bladder moves closer to the vagina posing the risk of increased radiation exposure of these critical normal structures during RT [4]. In the study by Pearcey et al. [9] an increase in treatment-related side effects with adjuvant radiotherapy was reported for patients undergoing surgery.

The potential for improved treatment outcomes with dose escalation warrants optimal normal tissue sparing under image guidance, and minimizing side effects of radiotherapy by decreasing normal tissue exposure is highly recommended in current radiotherapy practice [10]. To achieve this pertinent goal for endometrial cancer patients, SBRT emerged as a sophisticated treatment modality recently with promising results [6,7]. Besides delivering ablative radiation doses to small targets with hypofractionation, SBRT also offers homogeneous target dose distributions and improved critical organ sparing with great potential for dose escalation. Using this sophisticated technology with the dose and fractionation schemes identical to brachytherapy, dynamic-arc SBRT boost treatment after pelvic RT may be safely implemented with accurate set-up verification and without increasing normal tissue complications under image guidance [6].

Vaginal cuff boost brachytherapy has several limitations that may compromise the therapeutic outcomes. Since dose distribution is not homogeneous in brachytherapy, vaginal surface may be exposed to higher undesired radiation than the deeper mucosa. Clinical implementation of brachytrerapy clearly requires a dedicated brachytherapy unit, and continual exchange of expensive sources along with the dose inhomogeneities that may arise in irregularly shaped tumors or deep vaginal mucosal extention resulting in seriously compromised local control [7]. In both boost modalities, we used CT-compatible vaginal cylindrical applicator as a reference for the vaginal cuff irradiation and we planned the prescribed dose to the $4 \mathrm{~cm}$ of vaginal apex and to the depth of $5 \mathrm{~mm}$ around the cylinder for brachytherapy and $90 \%$ isodose line for SBRT to cover the PTV in all patients achieving homogeneous target volume dose distribution.

\section{Conclusion}

In this study, we compared 2-D brachytherapy boost and SBRT boost with the same dose and fractionation with respect to target, bladder and rectal point doses in endometrial cancer patients. Target point doses by both 3-D SBRT and 2-D Brachytherapy boost were detected to be equal at target, whereas OAR wise comparison for both modalities revealed statistically significant dose reduction in SBRT compared to brachytherapy (bladder $\mathrm{p}<0.001$, rectum $\mathrm{p}<0.001$ ). In conclusion, the use of SBRT modality conferred improved OAR sparing in vaginal cuff boost treatment for endometrial cancer without comprimising target point doses. Superior OAR sparing with SBRT may have implications for potential dose escalation to improve treatment outcomes in endometrial cancer management despite the need for futher supporting evidence.

\section{Acknowledgment}

None.

\section{Conflict of Interest}

There is no conflict of interest.

\section{References}

1. Charra-Brunaud C, Mazeron R (2013) Brachytherapy for endometrial cancers. Cancer Radiother 17(2): 106-110.

2. Creutzberg CL, van Putten WL, Koper PC, Lybeert ML, Jobsen JJ, et al. (2003) Survival after relapse in patients with endometrial cancer: results from a randomized trial. Gynecol Oncol 89 (2): 201209.

3. Mandell R, Nori D, Anderson L, Hılarıs B (1985) Postoperative vaginal radiation in endometrial cancer using a remote afterloading technique. Int J Radiat Oncol Biol Phys 11(3): 473-478.

4. Morrow CP, Bundy BN, Kurman RJ, Creasman WT, Heller P, et al. (1991) Relationship between surgical-pathological risk factors and outcome in clinical stage I and IIcarcinoma of the endometrium: a Gynecologic Oncology Group study. Gynecol Oncol 40 (1): 55-65. 


\section{Cancer Therapy \& Oncology International Journal}

5. MacLeod C, Fowler A, Duval P, D'Costa I, Dalrymple C, et al. (1999) Adjuvant high-dose rate brachytherapy with or without external beam radiotherapy post-hysterectomy for endometrial cancer. Int J Gynecol Cancer 9(3): 247-255.

6. Demiral S, Beyzadeoglu M, Uysal B, Oysul K, Kahya YE, et al. (2013) Evaluation of stereotactic body radiotherapy (SBRT) boost in the management of endometrial cancer. Neoplasma 60(3): 322-327.

7. Mollà M, Escude L, Nouet P, Popowski Y, Hidalgo A, et al. (2005) Fractionated stereotactic radiotherapy boost for gynecologic tumors: an alternative to brachytherapy? Int J Radiat Oncol Biol Phys 62(1) 118-124.

This work is licensed under Creative Commons Attribution 4.0 License

DOI: $10.19080 /$ CTOIJ.2017.07.555717
8. Baker DG, Krochak RJ (1989) The response of the microvascular system to radiation: a review. Cancer Invest 7(3): 287-294.

9. Pearcey RG, Petereit DG (2000) Post-operative high dose rate brachytherapy in patients with low to intermediate risk endometrial cancer. Radiother Oncol 56(1): 17-22.

10. Keys HM, Roberts JA, Brunetto VL, Zaino RJ, Spirtos NM, et al. (2004) A phase III trial of surgery with or without adjunctive external pelvic radiation therapy in intermediate risk endometrial adenocarcinoma a Gynecologic Oncology Group study. Gynecol Oncol 92(3): 744-751.

\section{Your next submission with Juniper Publishers} will reach you the below assets

- Quality Editorial service

- Swift Peer Review

- Reprints availability

- E-prints Service

- Manuscript Podcast for convenient understanding

- Global attainment for your research

- Manuscript accessibility in different formats

( Pdf, E-pub, Full Text, Audio)

- Unceasing customer service

Track the below URL for one-step submission https://juniperpublishers.com/online-submission.php 